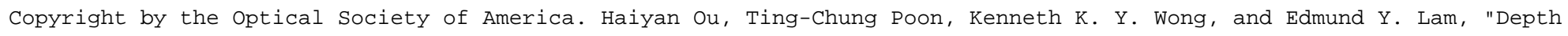

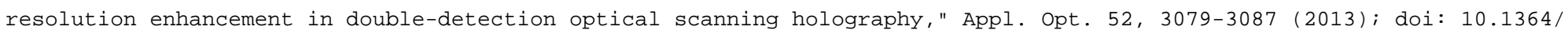
ao. 52.003079

\title{
Depth resolution enhancement in double-detection optical scanning holography
}

\author{
Haiyan Ou, ${ }^{1,2}$ Ting-Chung Poon, ${ }^{3,4}$ Kenneth K. Y. Wong, ${ }^{1}$ and Edmund Y. Lam ${ }^{1, *}$ \\ ${ }^{1}$ Department of Electrical and Electronic Engineering, University of Hong Kong, \\ Pokfulam Road, Hong Kong, China \\ ${ }^{2}$ Institute of Applied Physics, University of Electronic Science and Technology of China, Chengdu 610054, China \\ ${ }^{3}$ Bradley Department of Electrical and Computer Engineering, Virginia Tech, Blacksburg, Virginia 24061, USA \\ ${ }^{4}$ Shanghai Institute of Optics and Fine Mechanics (SIOM), Chinese Academy of Sciences, P.O. Box 800-211, \\ Shanghai 201800, China \\ *Corresponding author: elam@eee.hku.hk
}

Received 15 January 2013; revised 2 April 2013; accepted 2 April 2013; posted 3 April 2013 (Doc. ID 183469); published 29 April 2013

\begin{abstract}
We propose an optical scanning holography system with enhanced axial resolution using two detections at different depths. By scanning the object twice, we can obtain two different sets of Fresnel zone plates to sample the same object, which in turn provides more information for the sectional image reconstruction process. We develop the computation algorithm that makes use of such information, solving a constrained optimization problem using the conjugate gradient method. Simulation results show that this method can achieve a depth resolution up to $1 \mu \mathrm{m}$. (C) 2013 Optical Society of America

OCIS codes: (090.1995) Digital holography; (100.3190) Inverse problems; (100.3020) Image reconstruction-restoration; (110.1758) Computational imaging.

http://dx.doi.org/10.1364/AO.52.003079
\end{abstract}

\section{Introduction}

Optical scanning holography (OSH) is a technique that records the holographic information of a three-dimensional object on a two-dimensional hologram by lateral scanning [1-ㅣ] . It has many applications, ranging from microscopy to remote sensing [4-8]. In an OSH system, two coherent beams illuminate two pupil functions, respectively, with one commonly a point and the other a uniform pupil. They are then combined to take a lateral raster scan over a three-dimensional object. Subsequently, the interference of the two beams is detected by a single detector, resulting in an electronic hologram after some processing of the raw measurements [1]. Generally, the electronic hologram contains volume information of the object, i.e., multiple sections of this,

$1559-128 \mathrm{X} / 13 / 133079-09 \$ 15.00 / 0$

(C) 2013 Optical Society of America three-dimensional object. One important step is to obtain the individual section from the hologram, which is known as sectioning or sectional image reconstruction. The challenge of sectioning lies in the suppression of the defocus noise, which is the undesired residue signal of the other sections besides the one being reconstructed.

The conventional method for sectioning involves computing the convolution of the hologram with the conjugate impulse response at the focused section, but it suffers from large defocus noise from the unwanted sections [1]. There are many other ways to reduce the defocus noise, such as using inverse imaging [9-11], a Wiener filter [12], or the Wigner distribution [13]. Further development with inverse imaging also includes the use of edge-preserving regularization [14], edge detection to locate the sections [15], and compressed sensing [16]. A method that involves altering the system itself is to manipulate the pupil function of the OSH system [17]. Using 
two point pupils, with one as the positive spherical source and the other as the negative one, the defocus noise is reduced and the depth resolution can be doubled. Recently, to improve the depth resolution, a method using a dual-wavelength laser source has been proposed [18]. By switching the dualwavelength laser between 632 and $543 \mathrm{~nm}$, more information can be recorded, leading to a depth resolution of $2.5 \mu \mathrm{m}$.

In this paper, we propose another method to achieve better depth resolution by scanning the object twice at two different locations on the depth axis (i.e., $z$ axis), through which we can obtain two different sets of impulse responses to sample the same object. With proper image reconstruction schemes, we can have a much better depth resolution by combining the two holograms. Compared to the methods using two point pupils or dual-wavelength, which either requires modification of the OSH system or is restricted by the available laser source, the proposed system only requires controlling the location of the object on the $z$ axis, which is more cost effective and simple to implement.

\section{Double-Location Detection}

\section{A. System Setup}

The schematic of the system, which we will refer to as "double-detection OSH (DD-OSH)," is shown in Fig. 1. In this system, the laser source centered at frequency $\omega$ (or equivalently, wavelength $\lambda$ ) is divided into two paths by beam splitter BS1, in which one would pass through a pupil function $p_{1}(x, y)$, normally set to unity (i.e., $p_{1}(x, y)=1$ ) such that, after focusing by lens 1 , it would have a spherical wavefront on the object. Meanwhile, the other path out of the beam splitter would first have a frequency shift $\Omega$ via an acousto-optic frequency shifter (AOFS) and then arrive at another pupil function $p_{2}(x, y)$, commonly set to a delta function (physically a pinhole, with $p_{2}(x, y)=\delta(x, y)$ ), to become a plane wavefront on the object, after being collimated by lens 2 . The two coherent beams of different frequencies $\omega$ and $\omega+\Omega$ are then combined together by a second beam splitter

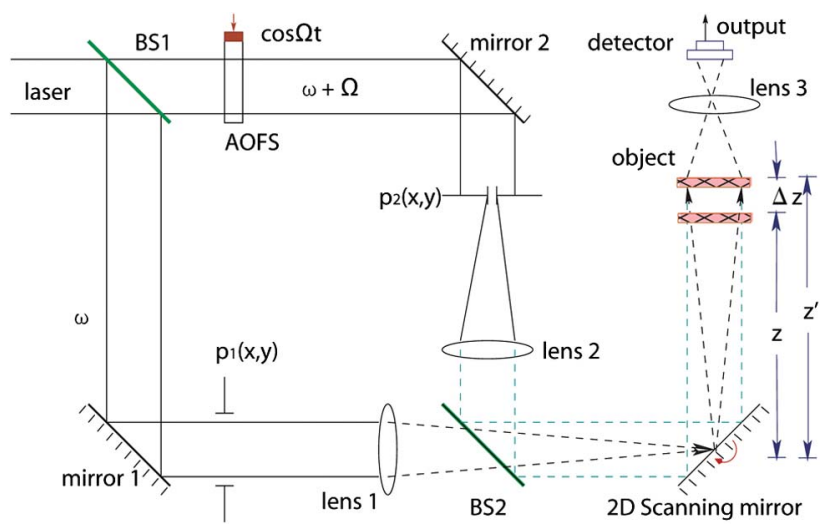

Fig. 1. Schematic of a DD-OSH system.
BS2, and are used to scan the object. The object is located at a distance $z$ away from the scanning mirror, as indicated in Fig. 1.

If we use $g(z, y ; z)$ to stand for the spatial impulse response of the Fresnel diffraction [1]

$$
g(x, y ; z)=\exp \left\{-j k_{0} z\right\} \frac{j k_{0}}{2 \pi z} \exp \left[-\frac{j k_{0}}{2 z}\left(x^{2}+y^{2}\right)\right],
$$

where $x, y, z$ are the coordinates, and $k_{0}=2 \pi / \lambda$ is the wavenumber of the optical source, with $\lambda$ representing the wavelength. Then, the optical field $C(x, y ; z)$ on the object can be expressed as [2]

$$
\begin{aligned}
C(x, y ; z)= & \hat{P}_{1}\left(\frac{k_{0} x}{f}, \frac{k_{0} y}{f} ; z\right) \exp \{j \omega t\} \\
& +\hat{P}_{2}\left(\frac{k_{0} x}{f}, \frac{k_{0} y}{f} ; z\right) \exp \{j(\omega+\Omega) t\},
\end{aligned}
$$

where $f$ is the focal length of the lens, and

$\hat{P}_{i}\left(\frac{k_{0} x}{f}, \frac{k_{0} y}{f} ; z\right)=\mathcal{F}\left\{p_{i}(x, y)\right\} * g(x, y ; z), \quad$ for $i=1,2$.

The symbol $*$ and $\mathcal{F}$ stand for the two-dimensional convolution operation and Fourier transform, respectively. A photodetector collects the transmitted and scattered light from the object and converts it into an electronic signal. Lens 3 is used to collected the light from the object. The optical transfer function of the whole system can thus be expressed as [2]

$$
\begin{aligned}
\mathcal{H}\left(k_{x}, k_{y} ; z\right)= & \exp \left[j \frac{z}{2 k_{0}}\left(k_{x}^{2}+k_{y}^{2}\right)\right] \\
& \times \iint p_{1}^{*}\left(x^{\prime}, y^{\prime}\right) p_{2}\left(x^{\prime}+\frac{f}{k_{0}} k_{x}, y^{\prime}+\frac{f}{k_{0}} k_{y}\right) \\
& \times \exp \left[j \frac{z}{f}\left(x^{\prime} k_{x}+y^{\prime} k_{y}\right)\right] \mathrm{d} x^{\prime} \mathrm{d} y^{\prime} .
\end{aligned}
$$

The principle and detailed mathematical analysis of the OSH system can be found in [2].

The description, so far, is identical to the conventional OSH system, but here is the difference: instead of scanning only once at each spatial location, in DD-OSH, it is scanned twice. This is achieved by moving the object to a second depth location, $z^{\prime}$, at a distance $\Delta z$ from $z$ (i.e., $z^{\prime}=z+\Delta z$ ). In so doing, we have doubled the data capture, which would provide more information for the reconstruction process. By combining the two holograms, we expect to have a better depth resolution for the sectioning process. However, one should note that a second scan means that the acquisition time is doubled. In addition, the object cannot move in between the two scans, making the method inappropriate for dynamic objects, if scanning is not fast enough. 


\section{B. DD-OSH Imaging Model}

The spatial impulse response of this DD-OSH system, also known as the Fresnel zone plates (FZPs), can be deduced from Eq. ( $\underline{4})$ with $p_{1}(x, y)=1$ and $p_{2}(x, y)=\delta(x, y)[\underline{2}]$ :

$$
h(x, y ; z)=-j \frac{1}{\lambda z} \exp \left\{j \frac{\pi}{\lambda z}\left(x^{2}+y^{2}\right)\right\},
$$

where $x, y$, and $z$ are the coordinates, and $\lambda$ is the wavelength of the optical source. Given an object with a complex amplitude $\psi(x, y ; z)$, its complex hologram generated by OSH can be expressed in a discrete form as [9]

$$
g(x, y)=\sum_{i=1}^{n}\left|\psi\left(x, y ; z_{i}\right)\right|^{2} * h\left(x, y ; z_{i}\right),
$$

where $z_{i}$ represents the depth location of the $i$-th section. We assume that there are a total of $n$ discrete sections.

To model the DD-OSH system, let us consider an object with two sections $(n=2)$ to simplify the description. When the object is placed in the first location, Eq. (6) can be written without the summation sign as

$$
\begin{aligned}
g_{1}(x, y)= & \left|\psi\left(x, y ; z_{1}\right)\right|^{2} * h\left(x, y ; z_{1}\right) \\
& +\left|\psi\left(x, y ; z_{2}\right)\right|^{2} * h\left(x, y ; z_{2}\right) .
\end{aligned}
$$

This can be expressed into a matrix form, with vectors $\boldsymbol{\psi}_{1}, \boldsymbol{\psi}_{2}$, and $\mathbf{g}_{1}$ representing $\left|\psi\left(x, y ; z_{1}\right)\right|^{2}$, $\left|\psi\left(x, y ; z_{2}\right)\right|^{2}$, and $g_{1}(x, y)$ using lexicographical ordering, respectively. Suppose the hologram is of size $N \times N$; then vectors $\boldsymbol{\psi}_{1}, \boldsymbol{\psi}_{2}$, and $\mathbf{g}_{1}$ are of length $N^{2}$. We then form two $N^{2} \times N^{2}$ matrices, $\mathrm{H}_{\mathrm{OSH}}\left(z_{1}\right)$ and $\mathrm{H}_{\mathrm{OSH}}\left(z_{2}\right)$ from $h\left(x, y ; z_{1}\right)$ and $h\left(x, y ; z_{2}\right)$, respectively, so that the convolution process in Eq. (7) becomes a matrix multiplication,

$$
\mathbf{g}_{1}=\mathrm{H}_{\mathrm{OSH}}\left(z_{1}\right) \boldsymbol{\psi}_{1}+\mathrm{H}_{\mathrm{OSH}}\left(z_{2}\right) \boldsymbol{\psi}_{2}+\mathbf{n}_{1}=\mathrm{H} \boldsymbol{\psi}+\mathbf{n}_{1} .
$$

We have added the term $\mathbf{n}_{1}$ above to denote the Gaussian random noise that exists in a typical imaging system and group the two terms together where

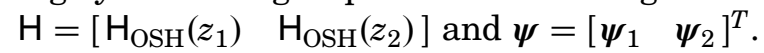

Next, we move the object away from the scanning mirror for the second scan by a distance $\Delta z$. The new distances of the two sections are

$$
z_{1}^{\prime}=z_{1}+\Delta z
$$

and

$$
z_{2}^{\prime}=z_{2}+\Delta z
$$

The complex hologram generated during the second scan can be expressed as

$$
\begin{aligned}
g_{2}(x, y)= & \left|\psi\left(x, y ; z_{1}^{\prime}\right)\right|^{2} * h\left(x, y ; z_{1}^{\prime}\right) \\
& +\left|\psi\left(x, y ; z_{2}^{\prime}\right)\right|^{2} * h\left(x, y ; z_{2}^{\prime}\right) .
\end{aligned}
$$

The corresponding matrix form is

$$
\mathbf{g}_{2}=\mathrm{H}_{\mathrm{OSH}}\left(z_{1}^{\prime}\right) \boldsymbol{\psi}_{1}+\mathrm{H}_{\mathrm{OSH}}\left(z_{2}^{\prime}\right) \boldsymbol{\psi}_{2}+\mathbf{n}_{2}=\mathrm{H}^{\prime} \psi+\mathbf{n}_{2},
$$

where, similar to the above, $\mathbf{n}_{2}$ is the Gaussian random noise for the second scan, and $\mathrm{H}^{\prime}=\left[\begin{array}{ll}\mathrm{H}_{\mathrm{OSH}}\left(z_{1}^{\prime}\right) & \mathrm{H}_{\mathrm{OSH}}\left(z_{2}^{\prime}\right)\end{array}\right]$. It should be noted that we have assumed that the object does not change between the two scans, and therefore $\left|\psi\left(x, y ; z_{1}^{\prime}\right)\right|^{2}=$ $\left|\psi\left(x, y ; z_{1}\right)\right|^{2}$ and $\left|\psi\left(x, y ; z_{2}^{\prime}\right)\right|^{2}=\left|\psi\left(x, y ; z_{2}\right)\right|^{2}$.

\section{Sectional Image Reconstruction}

To reconstruct object sections, we need to recover the object vector $\boldsymbol{\psi}$ given the measurements in $\mathbf{g}_{1}$ and $\mathbf{g}_{2}$, which is an inverse problem $[10,19]$. Due to its illposed nature $[20,21]$, we must use different methods to find an approximation for $\psi$ because of the regularization that must be imposed $[\underline{9}, 10]$. Considering the contribution of the two scans, we obtain the combined matrix formula

$$
\mathbf{g}=\left[\begin{array}{l}
\mathbf{g}_{1} \\
\mathbf{g}_{2}
\end{array}\right]=\left[\begin{array}{l}
\mathrm{H} \\
\mathrm{H}^{\prime}
\end{array}\right] \boldsymbol{\psi}+\left[\begin{array}{l}
\mathbf{n}_{1} \\
\mathbf{n}_{2}
\end{array}\right]=\mathrm{H}_{\mathrm{DD}} \boldsymbol{\psi}+\mathbf{n},
$$

where $\mathrm{H}_{\mathrm{DD}}$ now represents the overall DD-OSH system, and $\mathbf{g}$ is the entire observation data. One can see from Eq. (13) that an additional set of $N^{2}$ linear equations have been added to the same ill-posed problem due to the second scan.

The image reconstruction can be solved as a minimization problem such that the estimated signal $\boldsymbol{\psi}_{\text {est }}$ is given by [22-24]:

$$
\boldsymbol{\psi}_{\mathrm{est}}=\arg \min _{\boldsymbol{\psi}}\left\|\mathrm{H}_{\mathrm{DD}} \boldsymbol{\psi}-\mathbf{g}\right\|_{2}^{2}+\vartheta\|\mathrm{C} \psi\|_{2}^{2},
$$

where $\|\cdot\|_{2}$ denotes the $\ell_{2}$ norm, $\vartheta>0$ is a penalty parameter, and $C$ stands for the Laplacian operator. A more sophisticated regularization method has been shown to deliver better results $[14,25,26]$, but we choose this $\ell_{2}$-norm regularization for better comparison with the original inverse imaging for OSH [9] and the dual-wavelength technique [18]. The solution to Eq. (14) is

$$
\left(\mathrm{H}_{\mathrm{DD}}^{+} \mathrm{H}_{\mathrm{DD}}+\vartheta \mathrm{C}^{+} \mathrm{C}\right) \boldsymbol{\psi}_{\mathrm{est}}=\mathrm{H}_{\mathrm{DD}}^{+} \mathbf{g},
$$

where $\mathrm{H}_{\mathrm{DD}}^{+}$indicates the conjugate transpose of $\mathrm{H}_{\mathrm{DD}}$.

\section{Simulation and Analysis}

We analyze the sectioning results of the DD-OSH system with objects containing two and three sections. In addition to examining the visual quality, we also present analysis on the measurement error and the finite diameter of the apertures. 


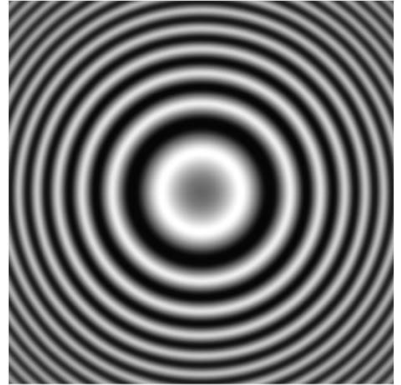

(a)

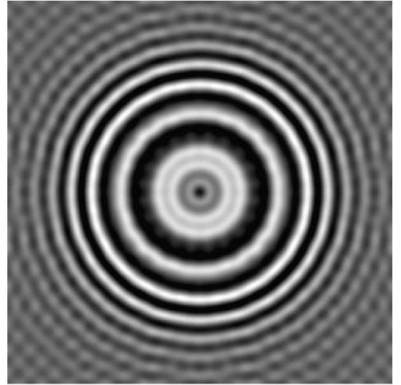

(b)
Fig. 2. (a) Real part of the ideal FZP of an OSH system and (b) real part of the FZP with the OSH simulator when $\mathrm{D}=40 \mathrm{~mm}$ and $f=50 \mathrm{~mm}$.

\section{A. Reconstruction with Two Sections}

Referring back to the system described in Fig. 1, we suppose that the object is illuminated by a HeNe laser, whose wavelength is $632 \mathrm{~nm}$. The two sections are located at $z_{1}=34 \mathrm{~mm}$ and $z_{2}=34.001 \mathrm{~mm}$, making them $1 \mu \mathrm{m}$ apart. Each object section has a size of $1 \mathrm{~mm} \times 1 \mathrm{~mm}$, which we discretize to a matrix size of $512 \times 512$. The two sections are scanned to generate the first hologram $\mathbf{g}_{1}$. We then move the object away from the scanning mirror by $\Delta z=15 \mathrm{~mm}$, such that $z_{1}^{\prime}=49 \mathrm{~mm}$ and $z_{2}^{\prime}=49.001 \mathrm{~mm}$. The objects are then scanned again, resulting in the second hologram $\mathbf{g}_{2}$.

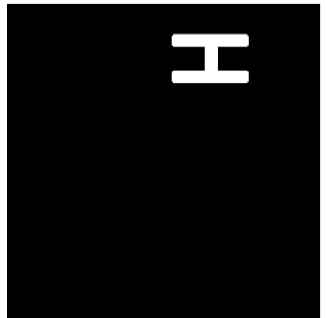

(a) section 1

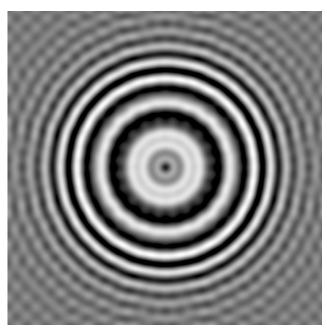

(c) real part of the FZP at $z_{1}$

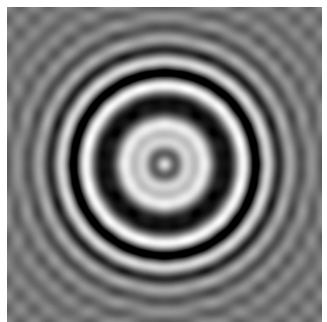

(e) real part of the FZP at $z_{1}^{\prime}$

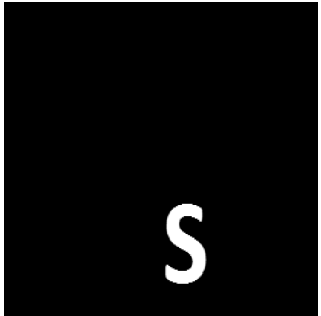

(b) section 2

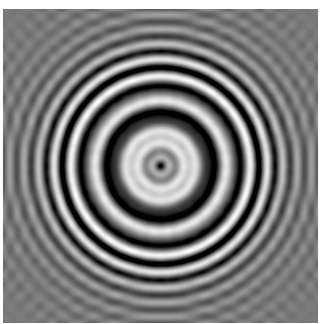

(d) real part of the FZP at $z_{2}$

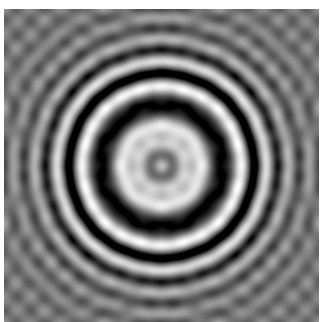

(f) real part of the FZP at $z_{2}^{\prime}$
Fig. 3. Two sections together with the real part of the FZPs for each scan.

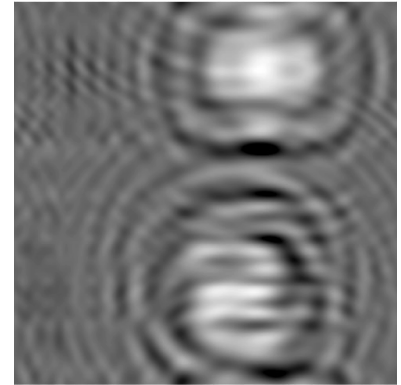

(a) real part of $g_{1}$

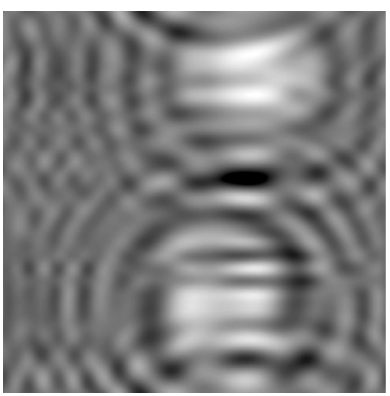

(c) real part of $g_{2}$

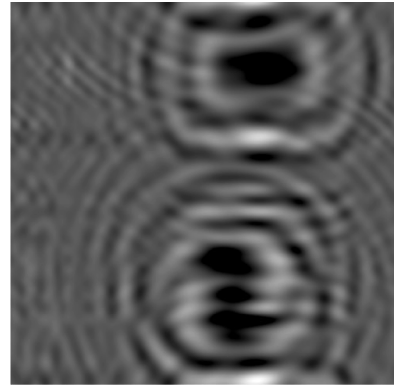

(b) imaginary part of $g_{1}$

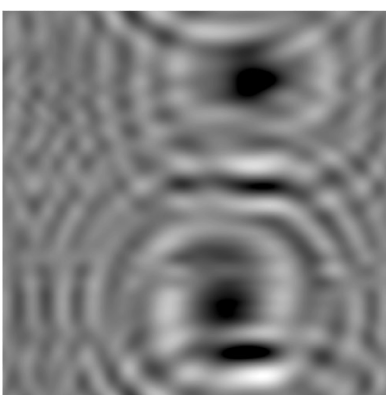

(d) imaginary part of $g_{2}$
Fig. 4. Holograms containing two sectional images of the object under double-location detection.

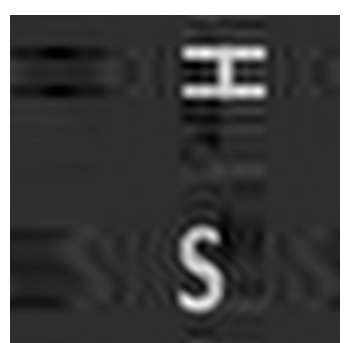

(a) $z_{1}$

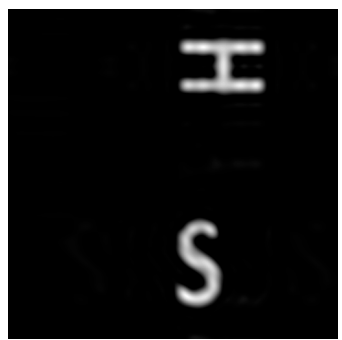

(c) $z_{1}$

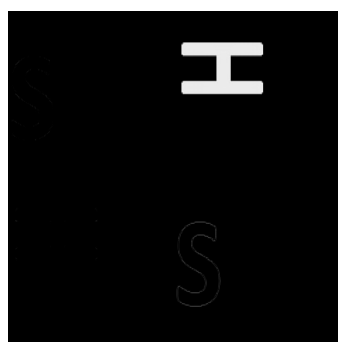

(e) $z_{1}$

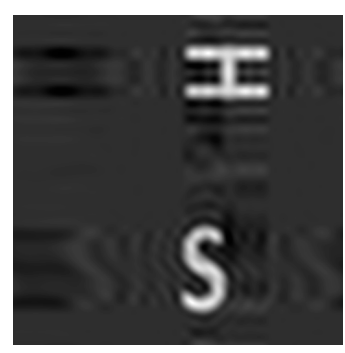

(b) $z_{2}$

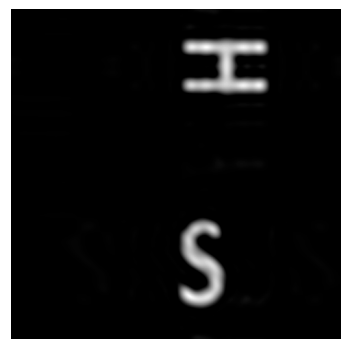

(d) $z_{2}$

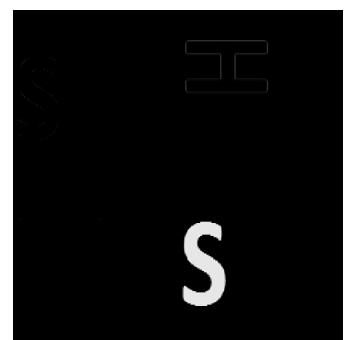

(f) $z_{2}$
Fig. 5. Sectioning results using (a),(b) the conventional method with SD-OSH; (c),(d) conjugate-gradient-based method with SD$\mathrm{OSH}$; (e),(f) conjugate-gradient-based method with DD-OSH. 


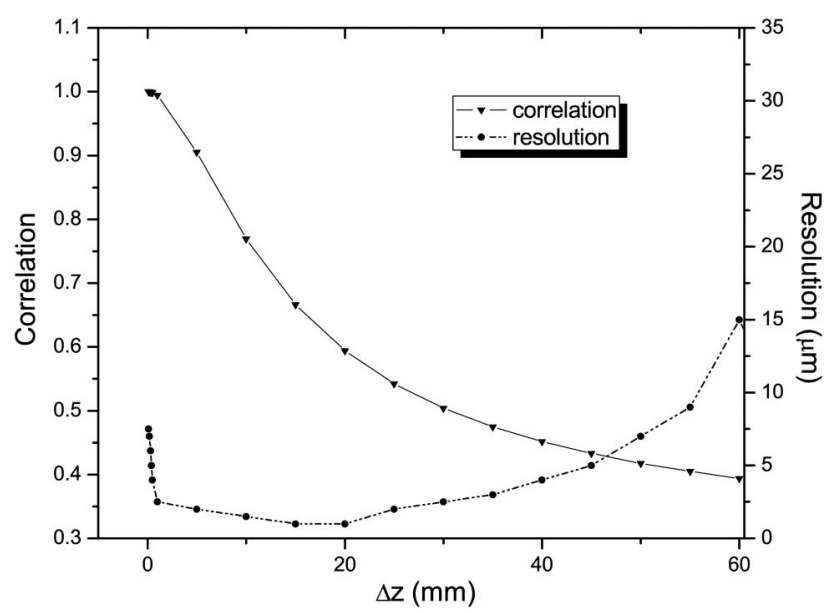

Fig. 6. Relationship between $\Delta z$ and resolution, and the relationship between $\Delta z$ and correlation of $\mathrm{H}$ and $\mathrm{H}^{\prime}$.

In order to evaluate the performance of the proposed method in a more precise system, we use an OSH simulator for the investigation. In the simulator, the finite diameter of the apertures together with the finite scanning step size of the scan process, and different kinds of noise are taken into consideration, the detail of which can be found in [18]. The system parameters are as follows: the pinhole aperture is $r=2.5 \mu \mathrm{m}$, the diameter of the collimated beam is $\mathrm{D}=40 \mathrm{~mm}$, and the focal length of the lens is $f=50 \mathrm{~mm}$. The scanning step size is $100 \mathrm{~nm}$. The thermal noise is a white Gaussian noise with an energy per bandwidth at $\sigma_{0}^{2}$, where $\sigma_{0}=12.75$. The Poisson noise has a Gaussian distribution $\mathcal{N}\left(\bar{p}, c \bar{p}^{2}\right)$ with $c=0.0025$, and $\bar{p}$ denotes the mean value of the detected hologram signal amplitude.

The real part of the FZP of an ideal OSH system and that of a realistic system using the simulator for the first section are shown in Fig. 2. One can see from this figure that the fringe patterns of the FZP using the simulator is different from the one in the ideal system. This is due to the fact that the latter case is using a numerical aperture, $\mathrm{NA} \approx D / 2 f$, less than that of the ideal case where $\mathrm{NA}=1$. Also, the other aperture is not an ideal pinhole but finite.

The two sections together with the real part of the FZPs for each scan are shown in Fig. 3. We can see that the FZP changes according to the distance from the scanning mirror. Comparing Figs. 3(c) with 3(e), or Figs. 3(d) with 3(f), we observe that as $z_{i}$ increases, the transition between opaque and transparent zones becomes slower, which indicates that the spatial sampling rate of these FZPs are slower. The complex holograms $g_{1}(x, y)$ and $g_{2}(x, y)$ are shown in Fig. 4 .

To reconstruct the two sections, we compare three methods: (1) conventional method with OSH data for one scan, which we call single-detection $\mathrm{OSH}$ (SD-OSH) hereafter, (2) conjugate-gradient-based reconstruction method with SD-OSH, and (3) conjugate-gradient-based method with DD-OSH. For SD-OSH, we simply use only the hologram $\mathbf{g}_{1}$ for reconstruction, as opposed to both $\mathbf{g}_{1}$ and $\mathbf{g}_{2}$ for DD-OSH. Figure $\underline{5}$ shows the reconstructed sections. We see that neither the conventional method nor the conjugate-gradient-based inverse imaging method provides acceptable sectioning results, because the depth of focus, which is approximately given by $2 \lambda /(\mathrm{NA})^{2}$, is $7.5 \mu \mathrm{m}$. The two sections are basically in focus simultaneously as evident from the results. On the other hand, with DD-OSH, the two sections are clearly separated, which indicates that the depth resolution in DD-OSH is equal to or smaller than $1 \mu \mathrm{m}$. The measured signal to the defocus noise ratio (SNR) is $37.17 \mathrm{~dB}$ in this case. For the discussions below, we define the depth resolution as the minimum sectional separation between image sections to retrieve an SNR equal to or better than this value.

The increase of the depth resolution is based on the additional information provided by the second scan, which is highly related to the displacement $\Delta z$ between the two scans. To quantify such information, we analyze both the relationship between $\Delta z$ and the depth resolution, as well as the relationship between $\Delta z$ and the correlation of the matrices $\mathrm{H}$ and $H^{\prime}$. The results are shown in Fig. 6 . First, we see that the correlation between the two matrices decreases as $\Delta z$ increases, which is to be expected because the FZPs are highly related to the axial distance. A larger $\Delta z$ leads to more changes in the FZPs, resulting in less similarity between $\mathrm{H}$ and $\mathrm{H}^{\prime}$. Second, we observe that the depth resolution of the DD-OSH system increases from 7.5 to $1 \mu \mathrm{m}$ as $\Delta z$ increases from 0.1 to $20 \mathrm{~mm}$. However, as $\Delta z$ goes beyond $20 \mathrm{~mm}$, the depth resolution would decrease. We conjecture that this is because as $z$ increases, the transition between the opaque and transparent zones of the FZPs is slower, which indicates that their spatial sampling rate becomes smaller (see Fig. 3 for

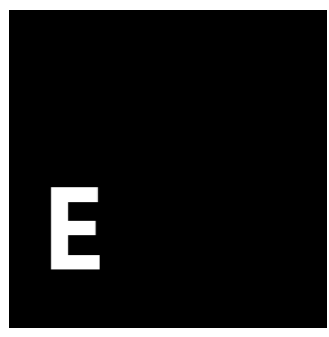

(a) section 3

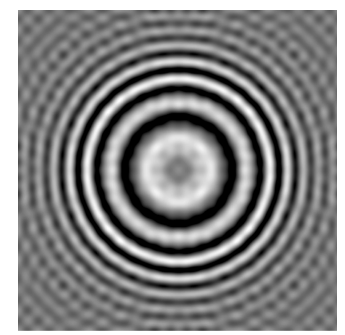

(b) real part of the FZP at $z_{3}$

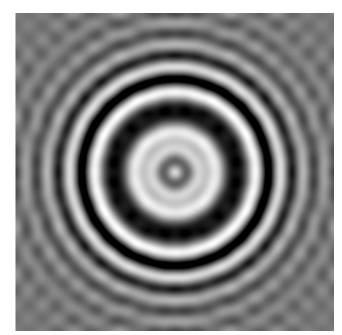

(c) real part of the FZP at $z_{3}^{\prime}$

Fig. 7. Third section together with the real part of the FZPs of each scan. 


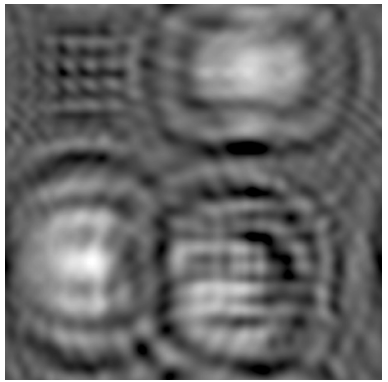

(a) real part of $g_{1}$

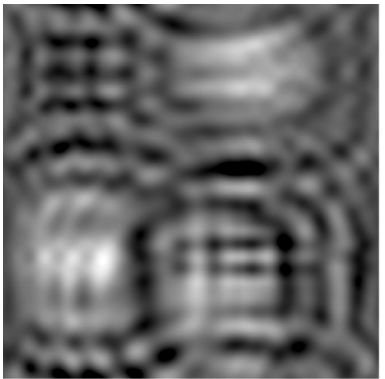

(c) real part of $g_{2}$

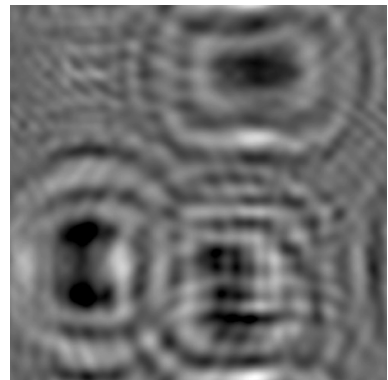

(b) imaginary part of $g_{1}$

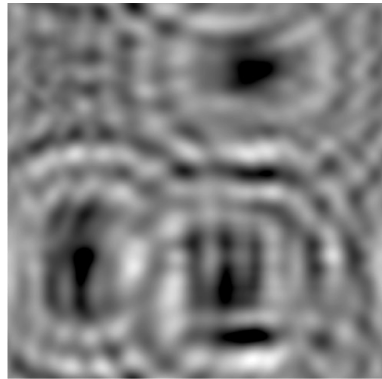

(d) imaginary part of $g_{2}$
Fig. 8. Holograms containing three sectional images of the object under double-location detection. reference). This means that less information can be captured. For the DD-OSH system, the enhancement of the depth resolution is based on the extra information of the object provided by the second scan. Therefore, there is a tradeoff between $\Delta z$ and the depth resolution. If $\Delta z$ is too small, then the transfer matrices $\mathrm{H}$ and $\mathrm{H}^{\prime}$ are highly correlated, which means less additional information can be obtained from the second scan. One extreme example is to set $\Delta z=0$, where the second set of data provides no extra information other than permitting noise reduction by averaging the two holograms. On the other hand, if $\Delta z$ is too big, the two matrices are less correlated, but the sampling rate of the FZPs becomes smaller as well. This would also result in less additional information from the second scan. One extreme example for this case is to set $\Delta z=\infty$, where no information would be generated during the second scan. In this case, we cannot expect to have any increase in the depth resolution.

\section{B. Reconstruction with Three Sections}

Next, we consider an object with three sections located at $z_{1}=34 \mathrm{~mm}, z_{2}=34.001 \mathrm{~mm}$, and $z_{3}=34.002 \mathrm{~mm}$, respectively. As such, the section

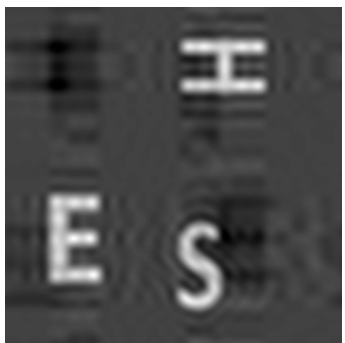

(a) $z_{1}$

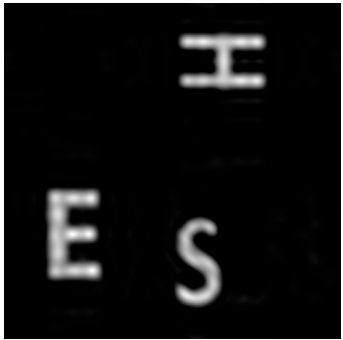

(d) $z_{1}$

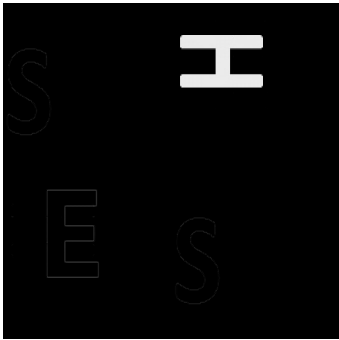

(g) $z_{1}$

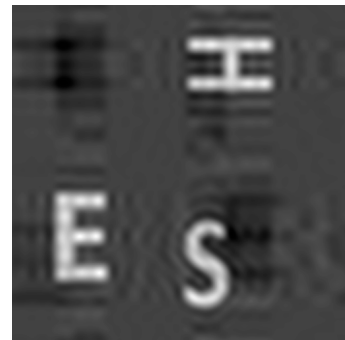

(b) $z_{2}$

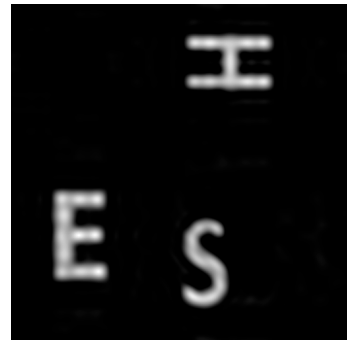

(e) $z_{2}$

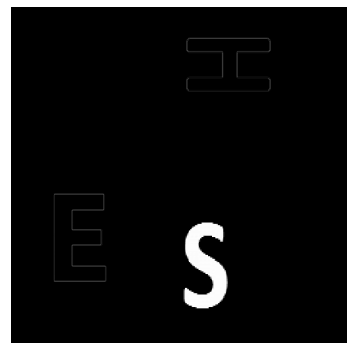

(h) $z_{2}$

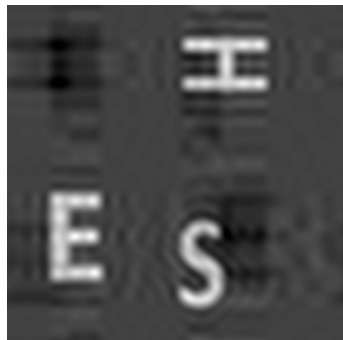

(c) $z_{3}$

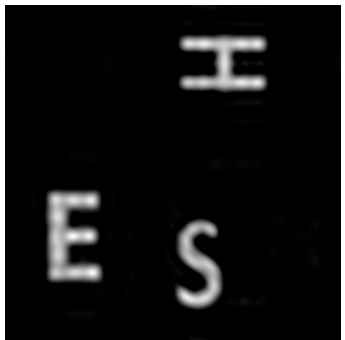

(f) $z_{3}$

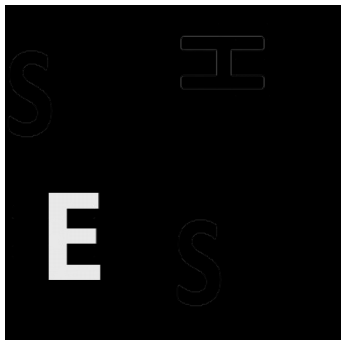

(i) $z_{3}$

Fig. 9. Sectioning results using (a)-(c) the conventional method with SD-OSH, (d)-(f) conjugate-gradient-based method with SD-OSH, and (g)-(i) conjugate-gradient-based method with DD-OSH. 
distance is again $1 \mu \mathrm{m}$. The experiment setup is the same as the one for the two-section object, as shown in Fig. 1. In this DD-OSH system, we also scan the object twice, with $\Delta z=15 \mathrm{~mm}$. The first two sections and the real part of the FZPs are the same as shown earlier in Fig. 3; the third section and the corresponding real part of the FZPs for the two scans are shown in Fig. 7.

The noisy holograms for each scan of this threesection object are shown in Fig. 8, which are more complex than the holograms for two-section objects. To reconstruct the three sections, we also compare the results from three different methods as above, namely, the former one, that is: (1) conventional method with SD-OSH measurements, (2) conjugategradient-based reconstruction method with SDOSH, and (3) conjugate-gradient-based method with DD-OSH. Figure 9 shows the reconstructed sections by the three different methods. We can see that the proposed DD-OSH method again outperform the other two methods in terms of suppressing signal from other sections.

\section{Measurement Error Analysis}

In the proposed DD-OSH system, we need to move the object along the $z$ axis between the two scans, causing inevitable errors in aligning the image data. In practice, the resolution of the available motorized translation stage is around $0.625 \mu \mathrm{m}$ (Zolix, TSA30C) which means the maximum error along the $z$ axis would be $0.625 \mu \mathrm{m}$. To test the effect of measurement error, we use the same two-section object with the proposed DD-OSH method, in which the object is moved away from the scanning mirror with $\Delta z=$ $15 \mathrm{~mm}$ for the second scan. However, as we incorporate the measurement error $\delta z$ along the $z$ axis, the object is actual moved $\Delta z^{\prime}$ away from the scanning mirror, where $\Delta z^{\prime}=\Delta z+\delta z$.

In the experiment, we test three different situations: (1) $\delta z=0 \mathrm{~mm}, z_{1}=34 \mathrm{~mm}, z_{2}=34.001 \mathrm{~mm}$; (2) $\delta z=0.625 \mu \mathrm{m}, z_{1}=34 \mathrm{~mm}, z_{2}=34.001 \mathrm{~mm}$; (3) $\delta z=0.625 \mu \mathrm{m}, z_{1}=34 \mathrm{~mm}, z_{2}=34.008 \mathrm{~mm}$. The sectioning results are shown in Fig. 10. One can observe from Figs. 10(a)-10(d) that as the measurement error increases, the defocus noise grows as well. When there is no measurement error (i.e., $\delta z=0$ ), the two sections are clearly separated; however, as the measurement error $\delta z$ increases to $0.625 \mu \mathrm{m}$, the sectioning results are too bad to distinguish the different sections. This indicates that the depth resolution decreases as the measurement error increases.

We then enlarge the section separation between the two sections to $8 \mu \mathrm{m} \quad\left(z_{1}=34 \mathrm{~mm}, z_{2}=\right.$ $34.008 \mathrm{~mm}$ ). The sectioning results with a measurement error of $\delta z=0.625 \mu \mathrm{m}$ and is shown in Figs. 10(e) and 10(f). One can observe that with a larger section separation, the measurement error $\delta z=0.625 \mu \mathrm{m}$ can be tolerated more. This indicates that the minimum section separation degrades to around $8 \mu \mathrm{m}$.

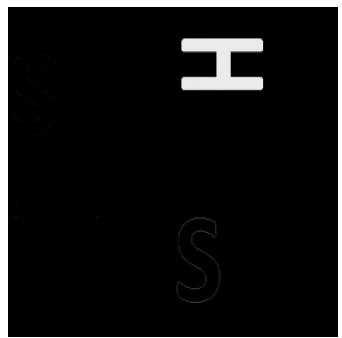

(a) $z_{1}$

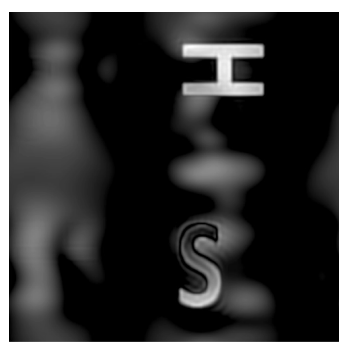

(c) $z_{1}$

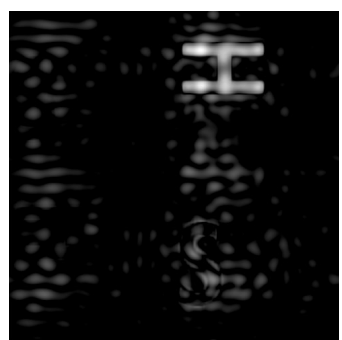

(e) $z_{1}$

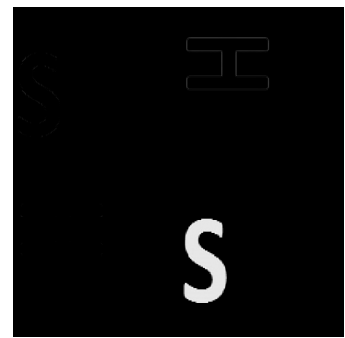

(b) $z_{2}$

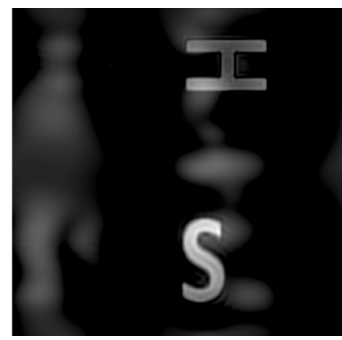

(d) $z_{2}$

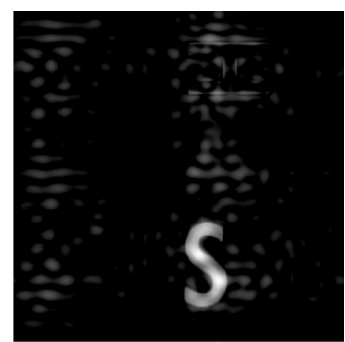

(f) $z_{2}$
Fig. 10. Sectioning results using the DD-OSH method with (a),(b) $\delta z=0 \mathrm{~mm}, z_{1}=34 \mathrm{~mm}, z_{2}=34.001 \mathrm{~mm} ; \quad$ (c),(d) $\delta z=$ $0.625 \mu \mathrm{m}, z_{1}=34 \mathrm{~mm}, z_{2}=34.001 \mathrm{~mm}$; (e),(f) $\delta z=0.625 \mu \mathrm{m}$, $z_{1}=34 \mathrm{~mm}, z_{2}=34.008 \mathrm{~mm}$.

We also analyze the relationship between the measurement error $\delta z$ and the depth resolution. The results are shown in Fig. 11. One can see that the depth resolution decreases as the measurement

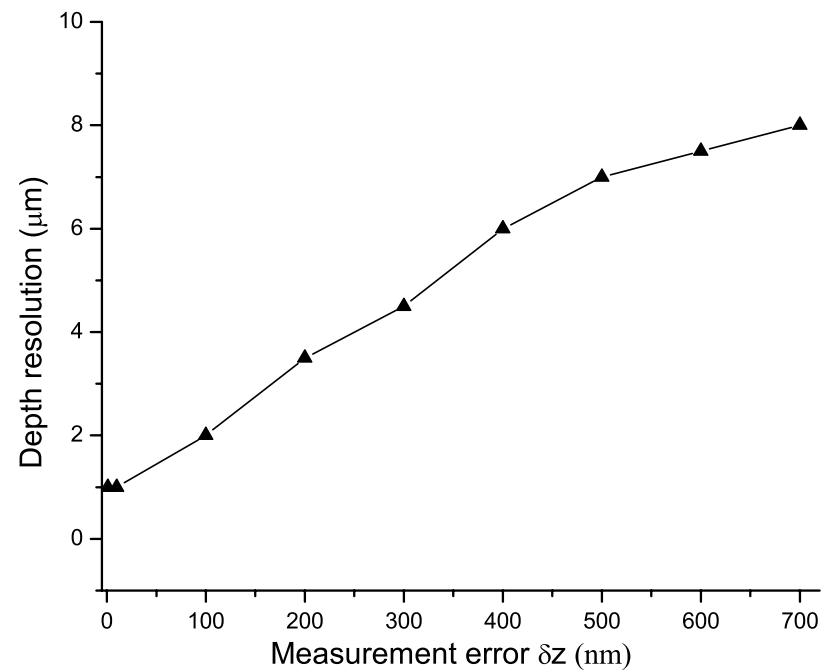

Fig. 11. Relationship between measurement error $\delta z$ and the depth resolution. 


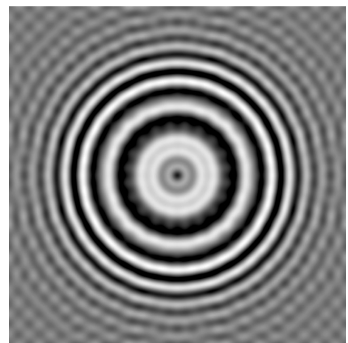

(a)

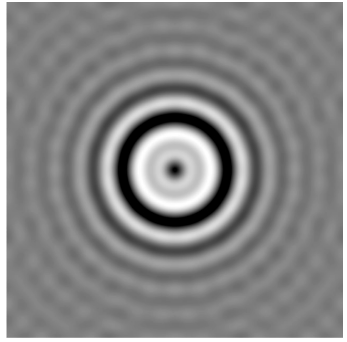

(d)

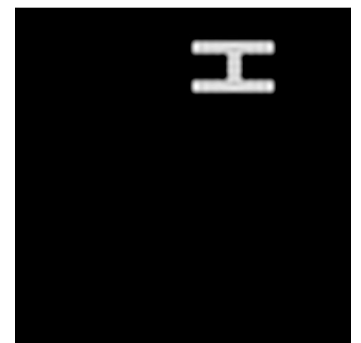

(b) $z_{1}$

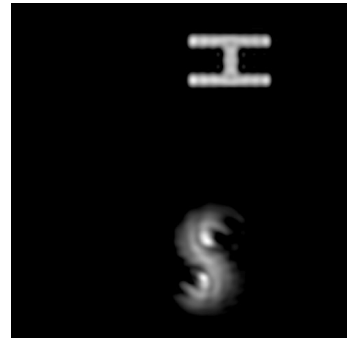

(e) $z_{1}$

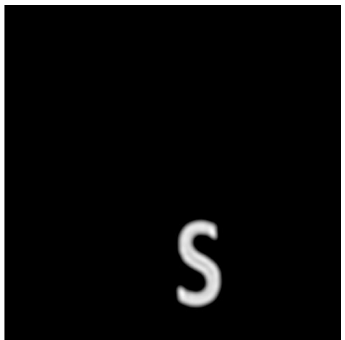

(c) $z_{2}$

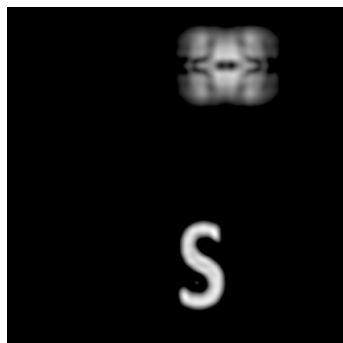

(f) $z_{2}$

Fig. 12. Real part of the FZPs and sectioning results using simulator with (a) - (c) $\mathrm{D}=40 \mathrm{~mm}$ and $f=50 \mathrm{~mm}$ and (d) - (f) $\mathrm{D}=25 \mathrm{~mm}$ and $f=50 \mathrm{~mm}$.

error $\delta z$ increases. When $\delta z$ reaches the maximum value $0.625 \mu \mathrm{m}$, the depth resolution would degrade to $8 \mu \mathrm{m}$.

\section{Analysis on the Finite Diameter of Aperture}

In a real OSH system, the FZPs of the OSH system are highly related to the finite diameter of the apertures, which in turn affect the sectioning results. We further investigate its effect with the simulator. In the simulation, both the diameter of the collimated beam $\mathrm{D}$ and the focal length of the lens $f$ are analyzed. The real part of the FZP for the first section and the sectioning results with a different collimated beam diameter are shown in Fig. 12. One can see that decreasing the diameter of the collimated beam $\mathrm{D}$ from 40 to $25 \mathrm{~mm}$ would result in more defocus noise in the sectioning process. This is also the case when increasing the focal length of the lens $f$ from 50 to $100 \mathrm{~mm}$, as can be seen in Fig. 13 .

It can be deduced from Figs. 12 and 13 that to improve the system performance we should use high numerical aperture in the optical setup. Further

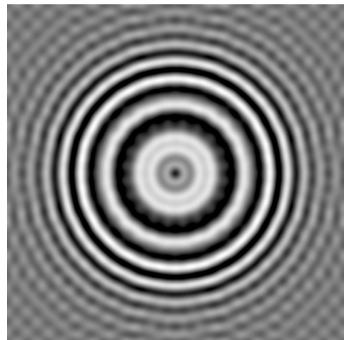

(a)

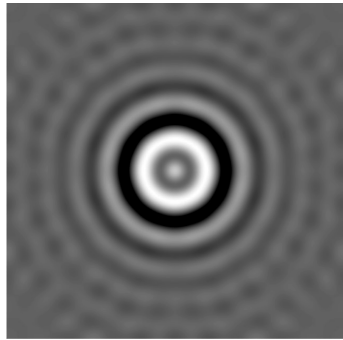

(d)

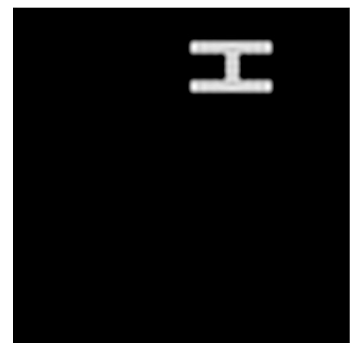

(b) $z_{1}$

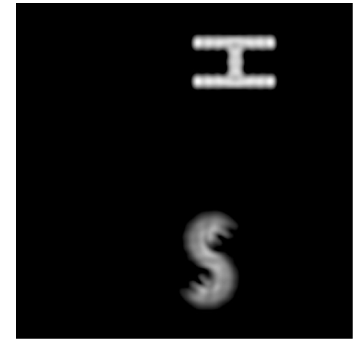

(e) $z_{1}$

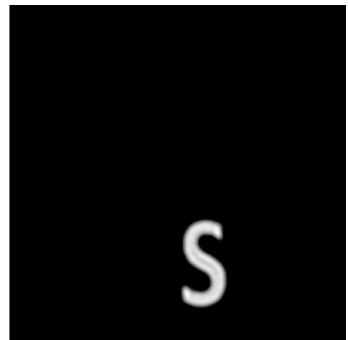

(c) $z_{2}$

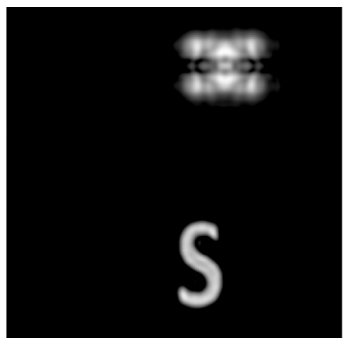

(f) $z_{2}$

Fig. 13. Real part of the FZPs and sectioning results using simulator with (a) - (c) $\mathrm{D}=40 \mathrm{~mm}$ and $f=50 \mathrm{~mm}$ and (d) $-(\mathrm{f}) \mathrm{D}=40 \mathrm{~mm}$ and $f=100 \mathrm{~mm}$. 
improvement can be achieved by increasing the sampling step size or the area for the recording holograms [18].

\section{Conclusions}

In the reconstruction of sectional images from a hologram generated by OSH, the difficulty lies in the suppressing of the defocus noise, i.e., the residual signals from the other sections. In this paper, we propose a way to increase the depth resolution with doublelocation detection. By moving the object away from the scanning mirror by $\Delta z$ and scanning for a second time, we can get two different sets of FZPs of the same object. Simulation results demonstrate that with proper values of $\Delta z$, the depth resolution can be increased up to $1 \mu \mathrm{m}$. It is worth noting that due to this double-location detection scheme, the total data acquisition time for the DD-OSH system is doubled compared to the SD-OSH system, which hinders its applications to in vivo imaging or applications in which the sample exposure time is strictly limited.

This work was supported in part by the Research Grants Council of the Hong Kong Special Administrative Region, China, under Projects HKU 7138/ $11 \mathrm{E}$ and $7131 / 12 \mathrm{E}$, and by the National Natural Science Foundation of China under Grant 61107018, as well as the Fundamental Research Funds for the Central Universities (ZYGX2011J033). T.-C. Poon also acknowledges the support by the Chinese Academy of Sciences Visiting Professorships for Senior International Scientists Program under Grant 2010T2G17.

\section{References}

1. T.-C. Poon, Optical Scanning Holography with MATLAB (Springer, 2007).

2. T.-C. Poon, "On the fundamentals of optical scanning holography," Am. J. Phys. 76, 738-745 (2008).

3. T.-C. Poon, "Optical scanning holography: origin, modern capabilities, and beyond," in OSA Topical Meeting in Digital Holography and Three-Dimensional Imaging (Optical Society of America, 2011), pp. DMA1.

4. J. Swoger, M. Martínez-Corral, J. Huisken, and E. H. K. Stelzer, "Optical scanning holography as a technique for high-resolution three-dimensional biological microscopy," J. Opt. Soc. Am. A 19, 1910-1918 (2002).

5. G. Indebetouw and W. Zhong, "Scanning holographic microscopy of three-dimensional fluorescent specimens," J. Opt. Soc. Am. A 23, 1699-1707 (2006).

6. T. Kim, T.-C. Poon, and G. Indebetouw, "Depth detection and image recovery in remote sensing by optical scanning holography," Opt. Eng. 41, 1331-1338 (2002).
7. T.-C. Poon, "Recent progress in optical scanning holography," J. Holography Speckle 1, 6-25 (2004).

8. T.-C. Poon, "Optical scanning holography-a review of recent progress,” J. Opt. Soc. Korea 13, 406-415 (2009).

9. X. Zhang, E. Y. Lam, and T.-C. Poon, "Reconstruction of sectional images in holography using inverse imaging," Opt. Express 16, 17215-17226 (2008).

10. E. Y. Lam, X. Zhang, H. Vo, T.-C. Poon, and G. Indebetouw, "Three-dimensional microscopy and sectional image reconstruction using optical scanning holography," Appl. Opt. 48, H113-H119 (2009).

11. X. Zhang, E. Y. Lam, T. Kim, Y. S. Kim, and T.-C. Poon, "Blind sectional image reconstruction for optical scanning holography," Opt. Lett. 34, 3098-3100 (2009).

12. T. Kim, "Optical sectioning by optical scanning holography and a Wiener filter," Appl. Opt. 45, 872-879 (2006).

13. H. Kim, S.-W. Min, B. Lee, and T.-C. Poon, "Optical sectioning for optical scanning holography using phase-space filtering with Wigner distribution functions," Appl. Opt. 47, D164D175 (2008)

14. X. Zhang and E. Y. Lam, "Edge-preserving sectional image reconstruction in optical scanning holography," J. Opt. Soc. Am. A 27, 1630-1637 (2010).

15. X. Zhang and E. Y. Lam, "Edge detection of three-dimensional object by manipulating pupil functions in optical scanning holography system," in Proceedings of IEEE International Conference on Image Processing (Institute of Electrical and Electronics Engineers, 2010), pp. 3661-3664.

16. X. Zhang and E. Y. Lam, "Sectional image reconstruction in optical scanning holography using compressed sensing," in Proceedings of IEEE International Conference on Image Processing (Institute of Electrical and Electronics Engineers, 2010), pp. 3349-3352.

17. G. Indebetouw, "Properties of a canning holographic microscope: improved resolution, extended depth-of-focus, and/or optical sectioning," J. Mod. Opt. 49, 1479-1500 (2002).

18. J. Ke, T.-C. Poon, and E. Y. Lam, "Depth resolution enhancement in optical scanning holography with a dual-wavelength laser source," Appl. Opt. 50, H285-H296 (2011).

19. C. R. Vogel, Computational Methods for Inverse Problems (SIAM, 2002).

20. A. N. Tikhonov and V. Arsenin, Solutions of Ill-Posed Problems (V.H. Winston \& Sons, 1977).

21. J. M. Blackledge, Digital Image Processing: Mathematical and Computational Methods (Horwood, 2005).

22. F. Natterer and F. Wübbeling, Mathematical Methods in Image Reconstruction (SIAM, 2001).

23. L. Vese, "A study in the BV space of a denoising-deblurring variational problem," Appl. Math. Optim. 44, 131-161 (2001).

24. G. Aubert and P. Kornprobst, Mathematical Problems in Image Processing: Partial Differential Equations and Calculus of Variations (Springer-Verlag, 2006).

25. H. Di, K. Zheng, X. Zhang, E. Y. Lam, T. Kim, Y. S. Kim, T.-C. Poon, and C. Zhou, "Multiple-image encryption by compressive holography," Appl. Opt. 51, 1000-1009 (2012).

26. D. J. Brady, K. Choi, D. L. Marks, R. Horisaki, and S. Lim, "Compressive holography," Opt. Express 17, 13040-13049 (2009). 